\title{
Urbanização, vulnerabilidade, resiliência: relações conceituais e compreensões de causa e efeito
}

\author{
Urbanization, vulnerability, resilience: conceptual relations and understanding of cause \\ and effect
}

Laíse do Nascimento Cabra|[a] [ㄱ, Gesinaldo Ataíde Cândido[a]

[a] Universidade Federal de Campina Grande (UFCG), Centro de Tecnologias e Recursos Naturais (CTRN), Campina Grande, PB, Brasil

Como citar: Cabral, L. N., \& Cândido, G. A. (2019). Urbanização, vulnerabilidade, resiliência: relações conceituais e compreensões de causa e efeito. urbe. Revista Brasileira de Gestão Urbana, 11, e20180063. https://doi.org/10.1590/21753369.011.002.A008

\section{Resumo}

Este artigo traz discussões inerentes às construções urbanas em um de seus cunhos mais abrangentes chamado de expansão urbana, fenômeno que se encontra inserido dentro do campo da urbanização e que tem causado transformações no espaço urbano. Tais transformações circundam as mazelas sociais e econômicas, desembocando em transtornos ao meio natural, formulando assim um histórico de vulnerabilidades socioambientais para o meio urbano. Diante desse contexto, este trabalho tem por objetivo propor relações de causa e efeito entre urbanização, vulnerabilidade e resiliência a partir da compreensão de riscos socioambientais decorrentes da intensificação do processo da expansão urbana. Define-se metodologicamente como um ensaio teórico respaldado pelas construções teóricas de alguns autores, dentre eles - Holling e Adger. Como resultado, tem-se cenários possíveis do que ocorre entre a complexidade desses fenômenos e a necessidade de aplicar tais conceitos à prática, ou seja, inferir empiricamente a relação desses conceitos com a realidade local de cada espaço urbano, cada um desses locais imbricados por suas realidades de identidade próprias, territorializando tais espaços e estabelecendo realidades próximas ou não da condição de urbano, vulnerável e resiliente.

Palavras-chave: Urbanização. Vulnerabilidade socioambiental. Resiliência. Cenários. Análise.

\section{Abstract}

This article permeates the discussions inherent to urban constructions in one of its most comprehensive ways called urban expansion, a phenomenon that is inserted within the field of urbanization and has caused transformations in urban space. These transformations surround the social and economic problems leading to disruption to the natural environment, thus formulating a history of social and environmental vulnerabilities to the urban environment. Given this context, this work proposes cause and effect study of the relationships between urbanization, vulnerability and resilience based on the understanding of social and environmental risks resulting from the intensification of the urban expansion process. It is possible, through theoretical-conceptual support, to know the capacities of answers and conflicts for urban spaces. It is defined methodologically as a theoretical essay supported by the theoretical constructions of some

LNC é geógrafa, mestra em Recursos Naturais, e-mail: laise.ufcg.rn@gmail.com

GAC é administrador, doutor em Engenharia de Produção, e-mail: gacandido@uol.com.br 
authors, among them - Holling, Adger. These pervade their theoretical discussions about urbanism, vulnerability, and resilience. As a result, it is necessary to apply these concepts to practice, that is, to empirically infer the relation of these concepts to the local reality of each urban space, each of these places imbricated by their own identity realities, territorializing such spaces and establishing realities whether or not they are urban, vulnerable and resilient.

Keywords: Urbanization. Socioenvironmental vulnerability. Resilience. Sceneries. Analysis.

\section{Introdução}

A complexidade dos fenômenos urbanização, vulnerabilidade e resiliência a serem abordados neste trabalho já é discutida há algumas décadas no meio científico. As dificuldades em resolver os problemas de ordem urbana, paisagística, do hábitat urbano e do meio urbano construído ocorrem desde a aceleração que modificou os meios de vida e do capital social decorrentes do processo de industrialização. $O$ fenômeno mais recentemente estudado é a resiliência. Há mais de duas décadas a resiliência tem sido estudada no meio acadêmico internacional em amplitudes de cenários de grandes pesquisas e hoje já fortalecida por diversos grupos e entidades acadêmicas.

Os três conceitos - urbanização, vulnerabilidade e resiliência - possuem formas de análises distintas, quando averiguados individualmente, e similitudes quando estudados e pesquisados conjuntamente. Há um consenso nos meios de pesquisa internacionais de que as separações que ocorreram entre esses fenômenos decorreram das construções teóricas e empíricas de seus pesquisadores e que foram colocadas e superpostas de modo superficial entre eles (Ostrom, 2002; Millennium Ecosystem Assessment, 2005; Janssen \& Ostrom, 2006; Adger et al., 2007).

O urbano tem sido um espaço de crescimento, desenvolvimento e contradições. É sabido que crescimento versus desenvolvimento são categorias de análise diferentes quando se trata dos assuntos sobre meio ambiente e economia, apesar de por vezes serem confundidas comumente em uma mesma vertente de análise. As questões econômicas são preponderantes em relação às questões ambientais. Ou seja, sempre que se fizer necessário escolher entre o crescimento econômico e a preservação, conservação ou manutenção do meio ambiente, os fatores que levam ao crescimento econômico serão a escolha notória.

Analisa-se que tais termos têm sofrido comparações errôneas quanto a suas definições. Essa pode ser colocada como uma das contradições marcantes e inerentes quando se trata do urbano ou do fenômeno da urbanização, pois o crescimento das cidades não tem alinho ao desenvolvimento urbano, e com isso causa o aumento da vulnerabilidade tanto em termos do hábitat humano quanto do ambiente construído, consequentemente diminuindo as capacidades de resiliência sociais e ecológicas.

Compreende-se que as capacidades sociais e econômicas no contexto urbano advêm das características de resiliência encontradas na comunidade, sendo e permanecendo como anseio de construção social e cultural e transformando-se no que Holling e Adger denominam por robustez do sistema. Ou seja, enxergam-se as potencialidades locais, o povo, suas características (muito além dos números e suportes estatísticos) e compõe-se o pensamento determinístico das funções reais, o vivido pelo habitante da cidade nesse perfil de estudo e análise.

Nessa linha de pensamento, este trabalho apresenta o desenvolver de uma conversa no tocante à exposição dos conceitos e nas diversas perspectivas de análise desses construtos - urbanização, vulnerabilidade e resiliência -, e assim tenta contribuir para uma análise contemporânea dos termos, lançando vertentes que busquem respostas para esse crescimento urbano que segue em todos os níveis de modo desestruturado.

Em meio a essas questões ou problemáticas inerentes a tais termos, este trabalho tem por objetivo propor relações teóricas e sistêmicas de causa e efeito entre urbanização, vulnerabilidade e resiliência a partir da compreensão de riscos socioambientais decorrentes da intensificação do processo da expansão 
urbana. Sendo possível, assim, obter um entendimento conceitual ao conhecer as capacidades de respostas e de conflitos para os espaços urbanos.

Tal objetivo está relacionado à intenção de compreender em quais esferas esses conceitos podem estar alinhados a fim de propiciar uma maior compreensão do fenômeno da urbanização nas suas múltiplas formas de relações de causa e efeito.

Além deste conteúdo introdutório e tendo por base a revisão de literatura, o artigo explicita na sua fundamentação teórica conteúdos relacionados ao fenômeno da urbanização e suas formas de crescimento sem infraestrutura, bem como uma análise sobre a vulnerabilidade e seus conceitos, definições e as perspectivas diante de uma vertente socioambiental urbana com seus elementos constituintes, e por fim uma compreensão conceitual sobre a resiliência. Com a união desses conceitos, pretende-se contribuir para uma nova discussão sobre a análise dos fenômenos de forma individual e conjunta diante dos desafios da vida contemporânea urbana.

\section{Aspectos metodológicos}

Em termos metodológicos, o estudo realizado pode ser classificado como um ensaio teórico respaldado pelas construções teóricas de autores que exploram os temas: urbanização, vulnerabilidade e resiliência.

A fim de tornar compreensível o caminho metodológico adotado nesta pesquisa, a Figura 1 demonstra o desenvolvimento da construção teórica dos temas apresentados, para assim construir uma análise mais precisa das complexidades entre as causas e efeitos desses fenômenos.

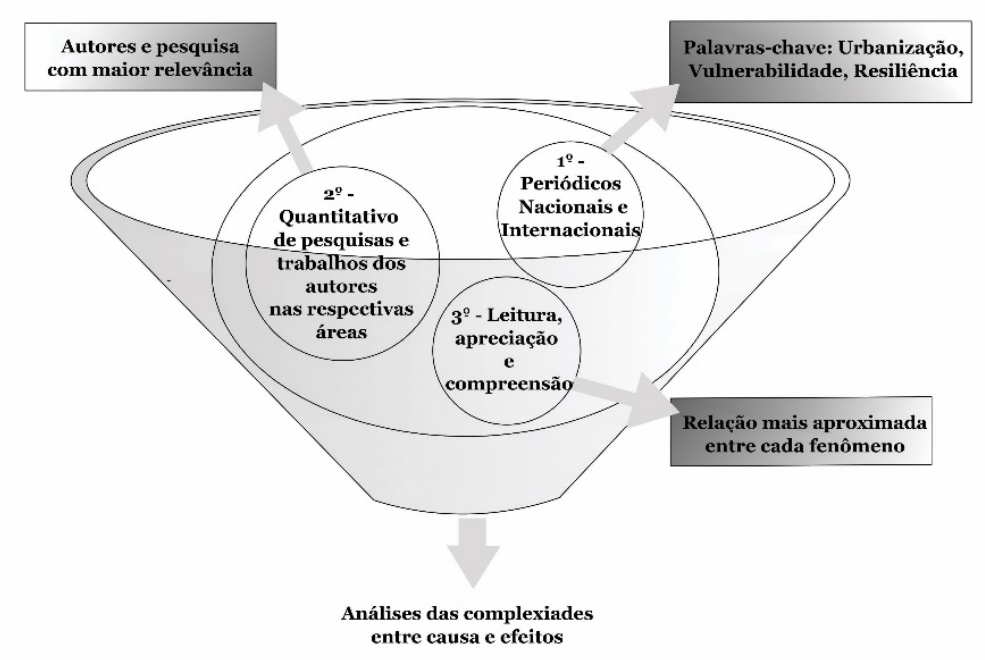

Figura 1 - Desenvolvimento da construção teórica dos temas. Fonte: Elaboração própria.

Desse modo, em um primeiro momento foi executada busca nos mais diversos periódicos internacionais e nacionais - de pesquisadores da área da urbanização, vulnerabilidade socioambiental e da resiliência, a fim de obter os resultados de análise teóricos objetivados - e assim estabelecer quais outros pesquisadores tinham seus nomes ligados/conectados aos três fenômenos: urbanização, vulnerabilidade e resiliência.

Já no segundo momento dessa busca teórica, analisou-se o contingente de trabalhos de cada autor, ou seja, avaliando o quantitativo de pesquisas e trabalhos desses nas respectivas áreas citadas. Por terceiro e último apontamento, foram separados para leitura, apreciação e compreensão os trabalhos que tinham uma relação mais aproximada entre cada fenômeno e quais poderiam dar uma melhor compreensão 
entre as análises de complexidades entre causa e efeito, aproximação ou distanciamento das vertentes dos fenômenos.

Ressalta-se a construção teórico-conceitual, devido às considerações permearem construtos teóricos de outros autores e pesquisadores, resultando na elaboração de uma nova perspectiva de análise quando da inter-relação desses três construtos com destaque para os problemas do urbano e suas correspondentes causas e efeitos.

\section{Fundamentação teórica}

\section{O fenômeno da urbanização}

O fenômeno da urbanização como processo histórico tem sido afiliado ao crescimento econômico, à diminuição dos índices de fertilidade, a uma maior expectativa de vida, à maior longevidade da população e aos deslocamentos geoespaciais. 0 avanço da técnica e da ciência fez com que a informação ganhasse impulso e velocidade como nunca dantes ocorrera e alcançando espaços de difícil acesso. 0 próprio fenômeno da urbanização e o seu decorrente processo de expansão urbana são fomentos e objetos dessa aceleração das tecnologias, principalmente como resultado do que trouxera a Revolução Industrial.

Em um mundo cada vez mais urbanizado onde o aumento da população tem se destacado em um cenário exponencial de crescimento e desigualdade, aumentam-se também as limitações de infraestrutura, saneamento, distribuição de água e o acesso aos serviços básicos de saúde e para manutenção da vida (UN, 2014a, 2014b). Percebe-se que o processo de crescimento das cidades não é somente desigual, mas que em cada lugar encontra-se um caminho próprio de construção no que tange às desigualdades, a exclusão das minorias - entende-se que as minorias têm se tornado um campo recorrente nas dicussões atuais, principalmente quando se retrata o eixo das desigualdades, e assim as dantes minorias ou os excludentes eclodem no espaço atual como maiorias.

Diante do pensamento de que mesmo em algumas ocasiões as similitudes dos problemas urbanos mais emergentes sejam aparentes em toda e qualquer cidade, entende-se que os problemas decorrentes e próprios do urbano são urgentes e apresentam questões que estão presentes em um pensamento globalizado e interconectado, em que a técnica, a informação e a ciência aperfeiçoam-se a todo instante e requerem soluções urgentes.

Segundo dados do relatório da United Nations - UN (2014a, 2014b) sobre a urbanização mundial, World Urbanization Prospects - WUP, as análises até então realizadas reforçam o dito anteriormente e projetam um crescimento da população mundial que vive em áreas urbanas, devendo este aumentar e atingir $66 \%$ até 2050 . Ressalta-se ainda, de acordo com esses dados, que $90 \%$ desse aumento serão concentrados em países da Ásia e da África, e que o crescimento populacional ao redor do mundo será de 2,5 bilhões de pessoas nas cidades, resultando em uma maior pressão e demanda sobre os recursos naturais, sociais e econômicos.

Na perspectiva desse crescimento, a vida urbana já foi tida como ideário, sendo associada a níveis mais elevados de educação, ao acesso aos serviços de saneamento básico e a oportunidades de emprego. No entanto, o crescimento rápido e desordenado prejudica as possibilidades de um desenvolvimento sustentável dessas estruturas urbanas, ocasionando exclusão, inchaço urbano e incapacidade de gerir os impactos causados pelas aglomerações populacionais.

Tais aspectos prejudicam e inviabilizam um planejamento urbano adequado e melhorias para a qualidade de vida urbana. Sem uma construção de planejamento sustentável ou com uma expansão urbana sem gerenciamento adequado, aumenta-se a probabilidade de crescimento desordenado expansão -, poluição e degradação ambiental, conjuntamente aos padrões insustentáveis de consumo. Além disso, sem uma infraestrutura adequada, ou quando as políticas públicas não são desenvolvidas para efetuar equitativamente uma melhor qualidade de vida para o habitante da cidade, os prejuízos 
socioeconômicos e ambientais tornam-se maiores. Sob esse ponto de vista, evidencia-se que o crescimento é diferente do desenvolvimento, onde em um determinado aspecto pode se ter crescimento e isto não significar o desenvolvimento social e ambiental daquele determinado espaço ou melhorias na percepção da qualidade de vida da população.

Desse ponto de vista apresentado, na Figura 2 tem-se um passo a passo ou desmembramento dessas fases de crescimento versus desenvolvimento no sentido de enxergar e compensar os ditames do progresso tecnológico que por vezes encontra-se acima das questões ambientais relativas à preservação/conservação ambiental, e nas próprias capacidades desse meio ambiente estar apto a sofrer as perturbações antrópicas e ambientais e vir a restabelecer equilíbrio entre consumo e disponibilidade, oferta e demanda. Dito isso, sempre no sentido de propiciar compreensão do que se tem disponível como matéria-prima, na transformação dessa matéria e na possibilidade de tê-la em uma escala temporal maior para outras gerações.

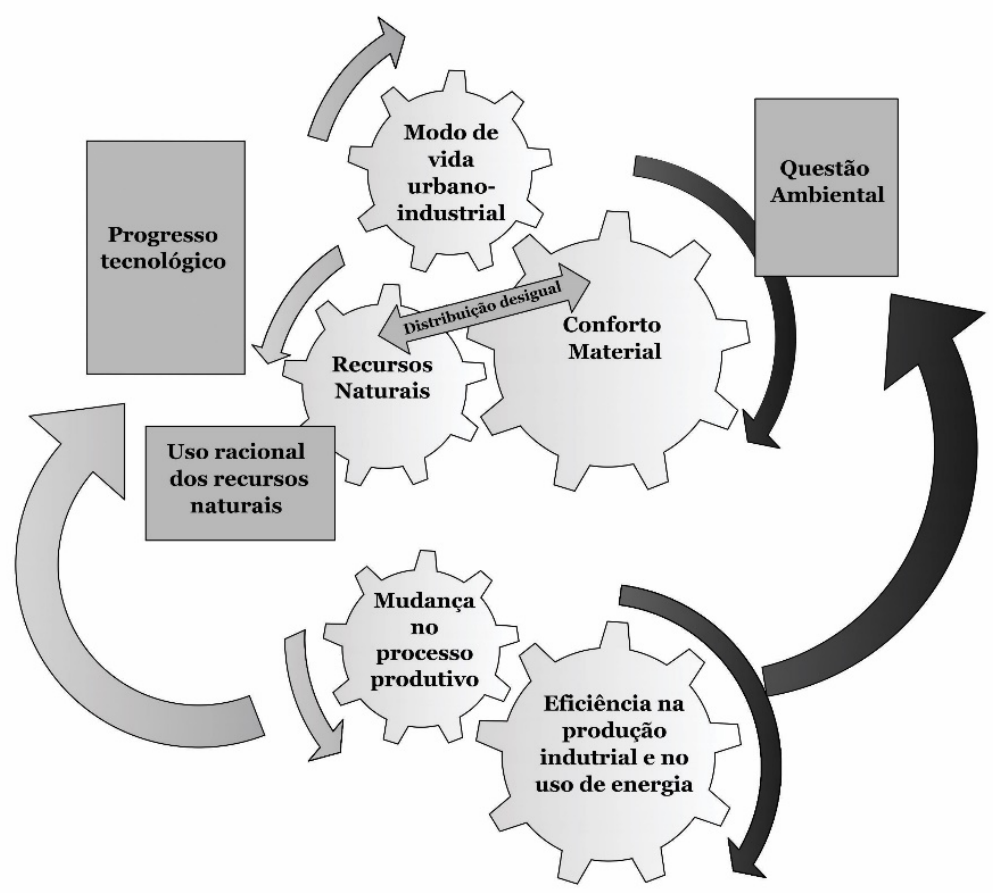

Figura 2 - Crescimento x Desenvolvimento em uma perspectiva urbano-industrial. Fonte: Elaboração própria.

Entende-se o exposto na Figura 2 como uma divisão que de um lado apresenta o progresso tecnológico com um modo de vida urbano-industrial, aliado a uma busca por mudanças que se apresentam recorrentes no processo produtivo. De outro, as questões ambientais relacionadas e relativizadas pelo uso racional dos recursos naturais e a necessidade de técnicas mais eficientes quanto à utilização de energias renováveis e tecnologias menos poluidoras dentro das indústrias e através da produção energética e da eficiência na produção industrial e no uso da energia.

Na parte central, a distribuição desigual decorrente desses processos é explicada pela busca por conforto material, e quando da utilização dos recursos naturais como matéria-prima a fim de uma transformação do recurso em produto. 0 que torna essa partilha desigual é justamente o fato de o crescimento da indústria e da expansão das cidades não acompanharem o ritmo de desenvolvimento e as estações do que se encontra no meio ambiente. As mudanças necessárias no espaço urbano para a construção e permanência das indústrias influenciam as cadeias da biodiversidade e no hábitat animal e humano, fases que demonstram como a vida urbana e o cotidiano são influenciados e assim modificados.

Ademais, ressalta-se que a urbanização e o seu então fenômeno da expansão urbana estão interligados aos pilares do desenvolvimento sustentável: econômico, social e ambiental. Compreende-se, então, que 
situações adversas decorrentes do processo de urbanização - construções de moradias em locais inadequados, por exemplo - são um agente vulnerável e que pode vir a causar vulnerabilidade, riscos e danos ao meio e ao homem como parte da natureza.

O tópico seguinte contribuirá no cerne do diálogo sobre a vulnerabilidade socioambiental e as diversas formas de conceituar, definir e algumas das diversas perspectivas que são encontradas para explicar essa conjuntura. A mesma se perfaz de cunho teórico-prático, pois se podem analisar empiricamente as questões que permeiam o que se propõe na abordagem teórica.

\section{Vulnerabilidade Socioambiental: conceitos, definições e perspectivas}

A vulnerabilidade tem sido analisada como um termo multidimensional por diversos pesquisadores. Essas diversas funções vêm das similitudes entre questões dos sistemas ecológicos, naturais e, de modo mais abrangente, socioambiental (Adger, 2006; Adger et al., 2007; Adger \& Hodbod, 2007; Smit \& Wandel, 2006). Nota-se também que não há consenso entre os autores das mais diversas áreas sobre sua conceituação. Ou seja, há diversas maneiras de enxergar conceituando, definindo e na busca de perspectivas para essa questão da qual muito tem sido relatada e observada no universo acadêmico.

De acordo com Adger (2006) e Adger et al. (2007), as formas de análise encontradas para os estudos em vulnerabilidade designaram encontros entre as ciências sociais e naturais. Além disso, relatam que as análises evidenciam que a vulnerabilidade é conceituada como sendo integrada a componentes que trazem exposição a perturbações, ao que também é descrito como choques ou estresses externos, a sensibilidade à perturbação e a capacidade adaptativa do meio.

Segundo Gallopín (2006), e dentro dessa perspectiva dos sistemas sociais e naturais, o autor sugere que a vulnerabilidade seja tratada como uma susceptibilidade para o meio ser ou estar traumatizado, oferecendo condições de restabelecimento (mudanças e transformações). Não seria então o resultado, mas as condições que foram dadas ao sistema.

Já para Turner et al. (2003a, 2003b), inerentes à vulnerabilidade estariam os riscos, sendo estes ameaças a um sistema onde é possível encontrar perturbações e estresses, sejam esses de níveis econômico, social, ecológico ou socioambiental (Berkes \& Folke, 1998).

Além dessas análises que o conceito de vulnerabilidade sofre, tem-se observado que há mudanças quanto à estrutura do sistema. Nesse diálogo, faz-se importante observar que deve haver uma discussão entre o que significa transformação (mudança) ou impacto no sistema quando se tenta definir vulnerabilidade socioambiental nos sistemas socioecológicos - SSEs (Gallopín et al., 1989; Van der Leeuw, 2001; Young et al., 2006). Esse pensamento reforça a tese de que não há conceituação pronta para vulnerabilidade, mas a proposição, um caminho. Fatos semelhantes aparecerão nas conversas mais adiante sobre resiliência.

Outro conceito que se relaciona com a vulnerabilidade é a exposição. Em geral, significa a duração ou medida de algum evento de estresse - perturbação (Adger, 2006; Adger et al., 2007; Kasperson et al., 2005). É o grau em que o meio socioecológico - uma cidade, por exemplo - suportará o evento estressor. É a forma do ecossistema urbano estar vulnerável a uma perturbação.

A partir dessa concepção de que a exposição é um componente da vulnerabilidade, um sistema que não esteja exposto a uma perturbação seria tomado por não vulnerável. Dada justamente a essa condição de suportar, passar pelo processo, evento, circunstância e posição.

A Figura 3 ilustra outro lado da vulnerabilidade, que seria uma maneira de indicar a capacidade de manter a estrutura do sistema contra perturbações, mesmo que a sua capacidade de resistência seja superada. Parece comum enxergar vulnerabilidade e resiliência como propriedades relacionadas de um Sistema Socioecológico - SSE. Mas a natureza específica da relação não é comum e nem óbvia. De acordo com Almeida (2010), o modelo teórico da Figura 3 é uma das maneiras de se traduzir e abordar o conceito de vulnerabilidade. 


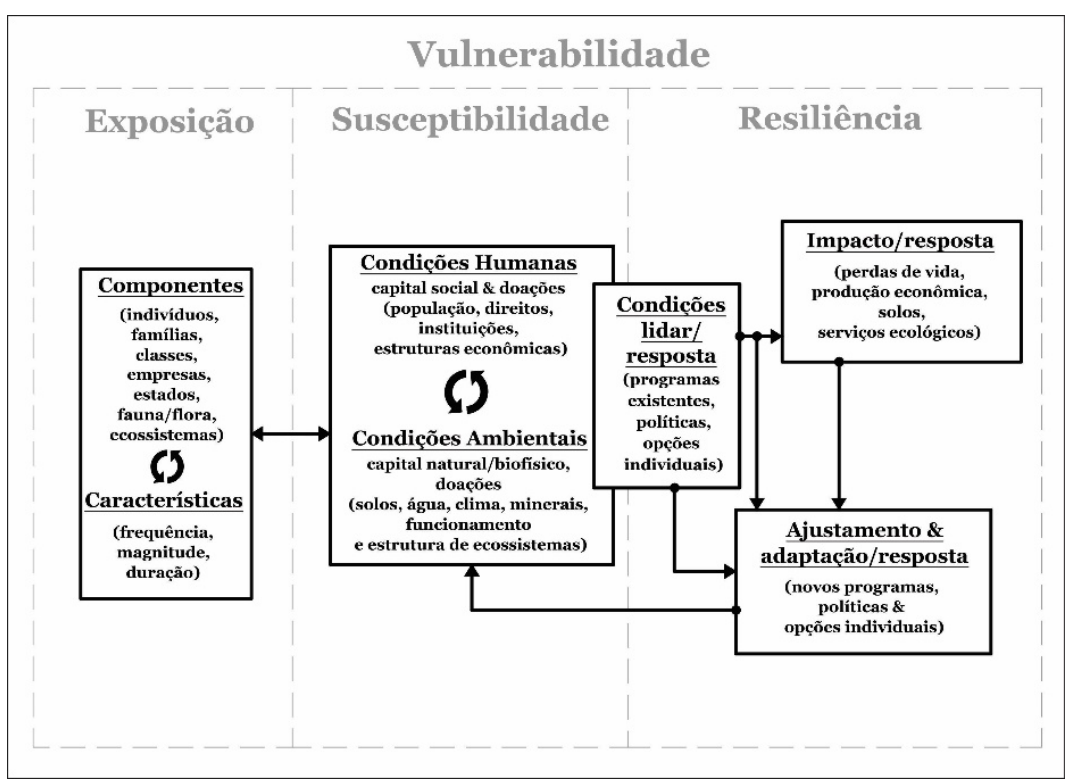

Figura 3 - Modelo estrutural da vulnerabilidade e detalhe para seus elementos constituintes. Fonte: Adaptado de Turner et al. (2003a) e Almeida \& Pascoalino (2009).

Configura-se, então, quando se trata do comportamento dos fatores que são integrantes da vulnerabilidade e de suas células constituintes, D'Ercole et al. (1994) e Almeida (2010) observam que sob esse ponto de vista na análise da vulnerabilidade o resultado pode ser uma sobreposição social ou espacial de seus componentes - os residentes nas cidades, seus bens materiais e a execução de trabalho.

Diante desses perfis de análise e compreensão da vulnerabilidade, o tópico seguinte enseja explicações sobre a conceituação sobre o fenômeno da resiliência, ao qual se entende que o ambiente urbano a ser pesquisado pode compreender as limitações entre a convivência do homem em congruência com a expansão urbana, a qual sufoca o ambiente natural, reduzindo-o e reprimindo esse.

Esta clareza acerca dos fenômenos estudados, com destaque para o ambiente urbanizado, torna-se pontual diante da gama de outras variáveis existentes para os estudos do urbano, da vulnerabilidade e da resiliência, fazendo com que a abordagem tomada compreenda um novo prisma para as atuais e futuras pesquisas, e o entendimento das particularidades de cada espaço estudado.

\section{O conceito de resiliência}

O conceito de resiliência teve como ponto de partida as discussões originadas no campo da ecologia e tornou-se reconhecido como parte do domínio dos sistemas sociais e ecológicos, ou como se tem nomeado: sistemas socioecológicos (SSEs). Adger (2000) introduz alguns conceitos em suas pesquisas sobre o fenômeno da resiliência, e uma dessas definições vem da expressão resiliência social. E a ela coloca-se como seus preceitos são encontrados no cotidiano, através das condições denominadas como estresses externos causados ao sistema. Tais perturbações causam mudanças nas análises dentro dos campos social, ambiental e político.

Para Holling $(1973,1985,1986)$, o conceito de resiliência está ligado à reação de um sistema após uma perturbação. Isto indica que não se refere a um resultado da ocorrência de algum estresse, mas da condição, da capacidade de reagir dessa comunidade, onde os aspectos sociais, culturais, econômicos e ambientais serão primordiais para o entendimento de uma possível reação que se daria igualmente a um processo de recuperação. Não se trata do que ocorre antes do problema, mas da solução que se espera diante dele.

Faz-se importante o alerta de que, para compreender a resiliência ou para que essa capacidade reativa ocorra, é necessário um histórico das exposições anteriores como forma de análise mais objetiva (Walker 
et al., 2004). Importa-se que seja entendido o que aconteceu antes como conflito na comunidade, bairro, rua ou qualquer espaço, antes do romper da perturbação. Pois, com a possibilidade de análise de situações de conflitos vividas anteriormente, pode-se compreender e efetivar um diagnóstico a fim de explicar o porquê da ocorrência sofrida e quais as possibilidades de enfrentar os desafios de reação no intuito da recuperação.

De acordo com a literatura e pesquisadores que versam sobre a resiliência, esta seria a condição de absorver determinada perturbação, conseguir adaptar-se e ainda manter suas funções de origem (Folke, 2006).

Adger (2000), ao discutir sobre resiliência social, define-a como a capacidade de as sociedades humanas suportarem perturbações externas comuns a sua infraestrutura social, como uma variabilidade social ou ambiental (ou socioambiental), da economia e como consequência da política.

Anderies et al. (2004), por sua vez, utilizam o conceito de robustez como uma das análises possíveis das características da resiliência. Este conceito seria capaz de discernir, objetivar e conhecer as flutuações desse sistema estudado, ou seja, como esse sistema consegue oscilar entre a estabilidade e a instabilidade do próprio sistema no qual está inserido. Para Price (2002) e Smit \& Wandel (2006), dentre essas condições especiais, notórias ou que sofrem destaque, encontra-se a capacidade adaptativa, também conhecida como capacidade de adaptação, e que demonstram esforços para explicar - talvez de uma forma ou modo ressignificado - o sentido de que a resiliência não norteia um sentido de estado confortável ou ideal, mas a possibilidade, a capacidade de voltar a uma situação anterior. Mesmo que esse estado anterior não seja estável (Carpenter et al., 2001; Nyström \& Folke, 2001; Price, 2002; Folke, 2006).

Assim, de acordo com os diversos referenciais estudados, a Tabela 1 mostra uma sequência de conceitos de resiliência a partir da interpretação mais estreita até a mais ampla com o contexto socioecológico.

Tabela 1 - Sequência de conceitos de resiliência

\begin{tabular}{|c|c|c|c|}
\hline Conceitos de Resiliência & Características & Focar em & Contexto \\
\hline Engenharia da Resiliência & Tempo de retorno, eficiência & Recuperação, constância & $\begin{array}{c}\text { Próximo de um equilíbrio } \\
\text { estável }\end{array}$ \\
\hline $\begin{array}{l}\text { Ecológico/resiliência dos } \\
\text { ecossistemas }\end{array}$ & $\begin{array}{l}\text { Capacidade tampão, resistir } \\
\text { a choques, manter a função }\end{array}$ & Persistência, robustez & $\begin{array}{c}\text { Equilíbrios múltiplos, } \\
\text { paisagens de estabilidade }\end{array}$ \\
\hline Resiliência socioecológica & $\begin{array}{c}\text { Ação recíproca do distúrbio } \\
\text { e reorganização, sustentar e } \\
\text { desenvolver }\end{array}$ & $\begin{array}{l}\text { Transformabilidade da } \\
\text { capacidade adaptativa, } \\
\text { aprendizagem, inovação }\end{array}$ & $\begin{array}{l}\text { Realimentação do sistema } \\
\text { integrado, dinâmicas } \\
\text { interações através da escala }\end{array}$ \\
\hline
\end{tabular}

Fonte: Adaptado de Carpenter et al. (2001), Folke (2006).

Compreende-se, a partir do exposto na Tabela 1, que a resiliência possui diversas características, focos, contextos e principalmente conceitos nas mais diversas áreas, como na engenharia, na psicologia e nas ciências ambientais.

A Tabela 1 expõe:

(1) a quantidade de estresse/distúrbio/pressão de que um sistema é capaz de absorver e ainda permanecer dentro do mesmo estado;

(2) a condição ou capacidade do sistema de se auto-organizar;

(3) a possibilidade do sistema de se reconstruir; e

(4) o aumento da capacidade de aprendizagem e adaptação.

Diante de tantas conceituações, a resiliência socioecológica é colocada e entendida pelas suas capacidades e potencialidades de recuperar-se e de se adaptar a novas situações de conflitos e estresses, bem como de voltar a um estágio inicial (Folke et al., 2002) - mesmo que danoso ou impróprio. Seria a condição de viver o desastre, mas não se apropriar de um contexto de desgraça ou aflição social, econômica e ambiental. Compreende-se que fatores como o de sensibilidade e robustez do sistema precisam ser levantados e levados em relevância como partes que fazem percepção e que têm um olhar 
sobre o todo, pois o estudo do todo também depende da análise das partes. 0 encaixe ou desencaixe socioambiental pode vir a ser a medida de verificação mais participante e homogênea para a resiliência.

\section{Resultados e discussões}

\section{A complexidade de estudos dos três fenômenos: urbanização, vulnerabilidade, resiliência}

Para estabelecer a relação entre esses três fenômenos, foi estabelecido um arcabouço teórico estudado a partir de Adger (2000), Adger \& Hodbod (2007), Holling (1973, 1996, 2004), Lucini (2014) e demais autores, e assim propor estabelecer a interdisciplinaridade das vulnerabilidades do processo de urbanização e entre os saberes sistêmicos da resiliência. Compreende-se que há uma relação no espaço urbano entre a urbanização e seu processo de expansão urbana, com a vulnerabilidade socioambiental e a resiliência socioecológica. A Figura 4 demonstra as relações de causa, efeito e perspectiva para a junção dos três fenômenos.

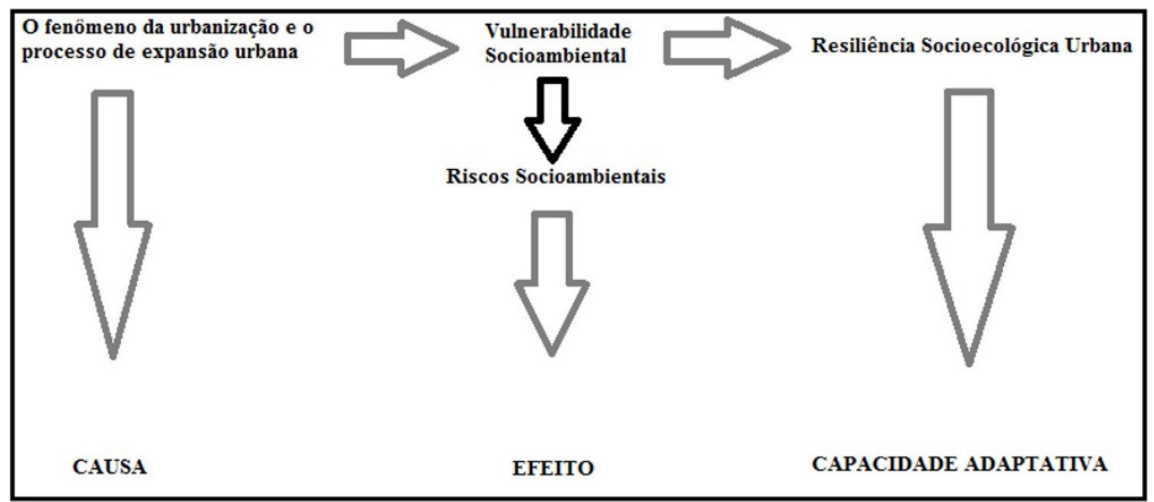

Figura 4 - Relações estabelecidas entre os fenômenos: expansão urbana, vulnerabilidade socioambiental e resiliência socioecológica urbana. Fonte: Elaboração própria.

Ainda sobre a Figura 4, o fenômeno da urbanização e o seu então processo de expansão urbana são tidos como causa para que, concomitantemente, ocorra o efeito da vulnerabilidade social e ambiental em determinado local, recaindo sobre situações de riscos socioambientais. Assim, a resiliência socioecológica urbana aparece como a possibilidade de averiguar qual a capacidade ou habilidade deste sistema urbano voltar ao seu estado original ou recuperar-se desta perturbação que lhe deixou vulnerável e que causou riscos. Tornando-o capaz de voltar a um estágio original ou de adquirir equilíbrio.

\section{Novas interpretações das análises conceituais dos fenômenos}

Como discutido nos itens anteriores, cada conceito tem sua própria forma de análise, de ser percebido e de se posicionar no espaço urbano. A análise parte agora de uma nova maneira de interpretação conceitual desses fenômenos. Entenda-se como um novo olhar diante das perspectivas da atualidade, do que se encontra como um problema diagnóstico da expansão das cidades, do aumento populacional e da falta de infraestrutura urbana. Essas são circunstâncias atreladas a essas questões, às quais, despretensiosamente, este trabalho se propõe a argumentar, não como resposta pronta e acabada, mas sim como forma de iniciar novos questionamentos a essas abordagens.

A Figura 5 expõe um organograma conceitual a fim de explicitar desde um estado inicial (inerte ou estático) a situação do meio antes de uma perturbação e como se dá todo esse processo, as etapas pelas quais se passa e as condições do meio urbano. 


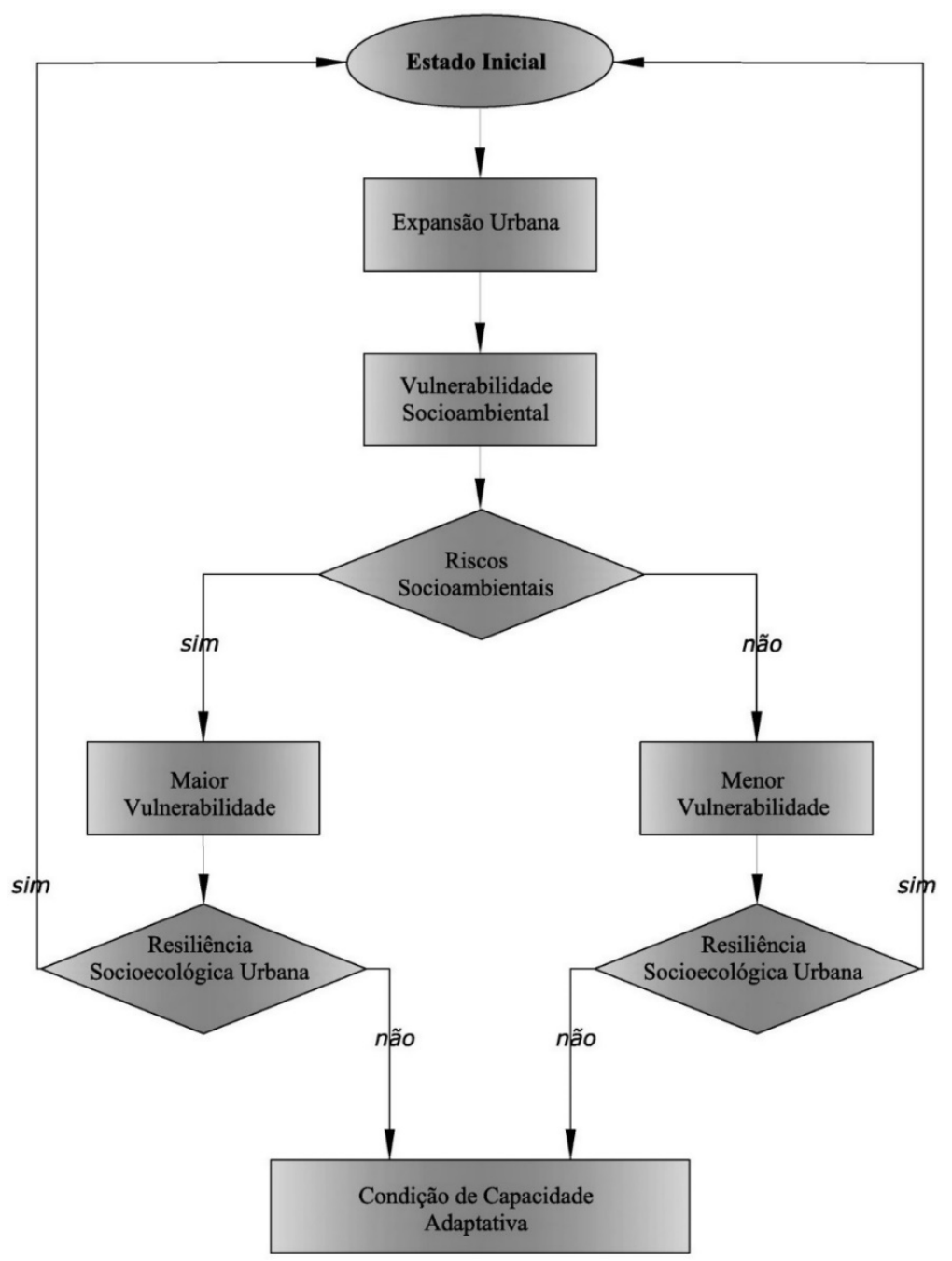

Figura 5 - Fluxograma das relações entre os fenômenos. Fonte: Elaboração própria.

O estado inicial é aquele em que a perturbação ainda não ocorreu. Seria uma situação vivida ou presenciada pelo meio e anterior ao choque (independentemente se esta circunstância seria confortável, favorável ou estável). Aqui não se avalia se o espaço possui uma situação de equilíbrio ou não, e sim a condição, capacidade ou possibilidade estabelecida para se voltar a esta condição, seja esta vulnerável ou resiliente.

Esse estado inicial passa pelos processos: primeiro, de expansão urbana, e depois de vulnerabilidade socioambiental. Esse encadeamento sugere que o fenômeno da urbanização introduzido pela expansão urbana ocasiona fragilidades que desembocam para vulnerabilidades no sistema biofísico ou econômicosocial-ambiental, conjuntamente, de modo que um gere influência no outro no campo das respostas às vulnerabilidades.

Quando apresentada a essa vulnerabilidade, concomitantemente vem a susceptibilidade aos riscos socioambientais. Se a resposta for sim à presença ou maior probabilidade de riscos socioambientais, então se tem uma maior vulnerabilidade. Diante desse pressuposto, procura-se estabelecer e analisar a possibilidade de aquele ecossistema urbano ser possuidor de capacidade de resiliência socioecológica urbana. Se sim a essa capacidade de resiliência, significa que aquele meio conseguiu superar o choque e voltar ao seu estado inicial, ou seja, anterior à perturbação sofrida pela vulnerabilidade ou a ponto de chegar a um estágio de recuperação desse trauma. Se a resposta encontrada à resiliência for não, induzse que esse sistema irá em busca de adaptar-se ao meio, o que temos expressado de condição para capacidade adaptativa. Talvez seja uma forma darwiniana de análise e pensamento, mas sugere a posição do homem, meio e dos objetos conseguirem superar ou não dificuldades no sentido do manter-se vivo. 
De estar ou manter-se além das expectativas e da ordem das coisas. Uma forma de subsistência do ecossistema urbano.

De outro modo (quando apresentadas as vulnerabilidades do sistema e a susceptibilidade dos riscos socioambientais), se a resposta for não a essas incidências, indica-se então uma menor vulnerabilidade. Diante desse estágio de menores condições aos riscos socioambientais, e, por conseguinte, menor vulnerabilidade, investiga-se a possibilidade de ocorrer ou não a resiliência socioecológica urbana. Compreende-se, até esse ponto, que se o lugar do urbano apresenta uma menor condição de vulnerabilidade socioambiental, entende-se que há possibilidade de diagnóstico positivo para a resiliência socioecológica urbana e uma resposta positiva (sim) de volta ao estado inicial. Caso a resposta encontrada seja negativa (não), sugere-se que esse hábitat urbano ainda poderá buscar uma condição para adaptar-se ao meio e sobreviver.

Ambas as situações indicam uma forte necessidade do meio de adaptarem-se às condições existentes. Explica-se. Mesmo em uma situação de maior ou menor vulnerabilidade, o hábitat urbano está sob algum perigo, sob o estado de ocorrência de algum risco socioambiental. Por isso, compreende-se de forma peculiar que a condição de capacidade adaptativa seria talvez a melhor proposição de restabelecimento da integridade do meio, da maneira de reensaiar-se na sua estrutura urbana e de apaziguar as dicotomias encontradas face à expansão desenfreada e desajustada perante a coisificação das pessoas nos próprios objetos urbanos.

\section{Considerações finais}

A ressignificação desses construtos faz parte das pesquisas recentes que cada vez mais têm comparado e estudado catástrofes socioecológica, as quais têm fragilizado o espaço urbano através do aumento da população, causando pressão aos recursos naturais e assim acarretando sistemas socioambientais vulneráveis, permitindo, ou não, uma capacidade de recuperação através da resiliência socioecológica urbana.

Este trabalho ensejou compreender como se dão as diferenças diante dos problemas cotidianos do urbano em face dos arranjos locais das cidades e sua pouca ou nenhuma capacidade de poder intervir em detrimento de uma infraestrutura urbana esfacelada e/ou inexistente.

Considera-se aqui uma oportunidade de observar o já dito. E de talvez conversar com pesquisadores da área sobre as diferentes abordagens conceituais nas diversas áreas de pesquisa, as quais alguns desses termos tiveram proporção. A exemplo, os diversos conceitos nas diferentes áreas de pesquisa para a resiliência. A condição de estudar e conceituar a vulnerabilidade como uma palavra de cunho e uso pessoal (na psicologia) ou estar alinhada aos termos e conceitos sobre riscos. Ademais, as definições dadas à urbanização, como se tem colocado na economia, geografia, arquitetura, engenharia e demais ramos de pesquisa.

Assim, a ciência continua a sua construção que se faz permanente e da qual dependem os questionamentos, argumentações e posições muitas vezes adversas. Parte-se de uma pergunta e a busca por respostas, as quais podem causar ainda mais inquietações e ter ainda mais em seus efeitos novas procuras aos problemas do cotidiano.

\section{Referências}

Adger, W. N. (2000). Social and ecological resilience: are they related? Progress in Human Geography, 24(3), 347364. http://dx.doi.org/10.1191/030913200701540465.

Adger, W. N. (2006). Vulnerability. Global Environmental Change, 16(3), 268-281.

http://dx.doi.org/10.1016/j.gloenvcha.2006.02.006. 
Adger, W. N., \& Hodbod, J. (2007). Ecological and social resilience. In G. Atkinson, S. Dietz \& E. Neumayer (Eds.), Handbook of sustainable development (pp. 78-90). Cheltenham: Edward Elgar Publishing.

http://dx.doi.org/10.4337/9781847205223.00012.

Adger, W. N., Agrawala, S., Mirza, M. M. Q., Conde, C., o'Brien, K., Pulhin, J., Pulwarty, R., Smit, B., \& Takahashi, K. (2007). Assessment of adaptation practices, options, constraints and capacity. In M. L. Parry, O. F. Canziani, J. P. Palutikof, C. E. Hanson, P. J. van der Linden (Eds.), Climate change 2007: impacts, adaptation and vulnerability. contribution of working group ii to the fourth assessment Report of the Intergovernmental Panel on Climate Change (pp. 719-743). Cambridge: Cambridge University Press.

Almeida, L. D., \& Pascoalino, A. (2009). Gestão de risco, desenvolvimento e (meio) Ambiente no Brasil-um estudo de caso sobre os desastres naturais de Santa Catarina. In Simpósio Brasileiro de Geografia Física Aplicada (pp. 13). Viçosa: UFV.

Almeida, L. Q. D. (2010). Vulnerabilidades socioambientais de rios urbanos: bacia hidrográfica do rio Maranguapinho, Região metropolitana de Fortaleza, Ceará (Tese de doutorado). Instituto de Geociências e Ciências Exatas, Universidade Estadual Paulista, Rio Claro.

Anderies, J. M., Janssen, M. A., \& Ostrom, E. (2004). A framework to analyze the robustness of social-ecological systems from an institutional perspective. Ecology and Society, 9(1), art18. http://dx.doi.org/10.5751/ES-00610-090118.

Berkes, F., \& Folke, C. (1998). Linking social and ecological systems for resilience and sustainability. In F. Berkes, C. Folke \& Colding, J., (Eds.), Linking social and ecological systems: management practices and social mechanisms for building resilience (pp. 1-25.). Cambridge: Cambridge University Press.

Carpenter, S., Walker, B., Anderies, J. M., \& Abel, N. (2001). From metaphor to measurement: resilience of what to what? Ecosystems (New York, N.Y.), 4(8), 765-781. http://dx.doi.org/10.1007/s10021-001-0045-9.

D’Ercole, R., Thouret, J.-C., Dollfus, O., \& Asté, J.-P. (1994). Les vulnérabilités des sociétés et des espaces urbanisés: concepts, typologie, modes d'analyse. Revue de Geographie Alpine, 82(4), 87-96. http://dx.doi.org/10.3406/rga.1994.3776.

Folke, C. (2006). Resilience: the emergence of a perspective for social-ecological systems analyses. Global Environmental Change, 16(3), 253-267. http://dx.doi.org/10.1016/j.gloenvcha.2006.04.002.

Folke, C., Carpenter, S., Elmqvist, T., Gunderson, L., Holling, C. S., \& Walker, B. (2002). Resilience and sustainable development: building adaptive capacity in a world of transformations. Ambio, 31(5), 437-440.

http://dx.doi.org/10.1579/0044-7447-31.5.437. PMid:12374053.

Gallopín, G. C. (2006). Linkages between vulnerability, resilience, and adaptive capacity. Global Environmental Change, 16(3), 293-303. https://doi.org/10.1016/j.gloenvcha.2006.02.004.

Gallopín, G. C., Gutman, P., \& Maletta, H. (1989). Global impoverishment, sustainable development and the environment: a conceptual approach. International Social Science Journal, 41(121), 375-397.

Holling, C. (2004). From complex regions to complex worlds. Ecology and Society, 9(1), art11. http://dx.doi.org/10.5751/ES-00612-090111.

Holling, C. S. (1973). Resilience and stability of ecological systems. Annual Review of Ecology and Systematics, 4(1), 1-23. https://doi.org/10.1146/annurev.es.04.110173.000245.

Holling, C. S. (1985). Perceiving and managing the complexity of ecological systems. In S. Aida, H. Allen, K. E. Atlan (Eds.), The science and praxis of complexity: contributions to the symposium held (at Montpellier, France, May 9-11, 1984) (pp. 217-227). Tokyo: United Nations University Press.

Holling, C. S. (1986). The resilience of terrestrial ecosystems: local surprise and global change. In: W. Clark, C., \& R. E. Munn (Eds.), Sustainable development of the biosphere (pp. 291-321). Cambridge: Cambridge University Press.

Holling, C. S. (1996). Surprise for science, resilience for ecosystems, and incentives for people. Ecological Applications, 6(3), 733-735. http://dx.doi.org/10.2307/2269475.

Janssen, M. A., \& Ostrom, E. (2006). Empirically based, agent-based models. Ecology and Society, 11(2), art37. http://dx.doi.org/10.5751/ES-01861-110237. 
Kasperson, R. E., Dow, K., Archer, E., Caceres, D., Downing, T., Elmqvist, T., Eriksen, S., Folke, C., Han, G., Iyengar, K., Vogel, C., Wilson, K., \& Ziervogel, G. (2005). Vulnerable people and places. In R. Hassan, R. Scholes \& N. Ash (Eds.), Ecosystems and human well-being: current state and trends (Vol. 1, pp. 143-164). Washington: Island Press.

Lucini, B. (2014). Disaster resilience from a sociological perspective: exploring three italian earthquakes as models for disaster resilience planning (Humanitarian Solutions in the 21st Century). USA: Springer International Publishing. https://doi.org/10.1007/978-3-319-04738-6.

Millennium Ecosystem Assessment. (2005). Ecosystems and human well-being: synthesis (Vol. 5, 155 pp., The Millennium Ecosystem Assessment series). Washington: Island Press.

Nyström, M., \& Folke, C. (2001). Spatial resilience of coral reefs. Ecosystems (New York, N.Y.). http://dx.doi.org/10.1007/s10021-001-0019-y.

Ostrom, E. (2002). Panarchy: understanding transformations in human and natural systems: Lance H. Gunderson and C.S. Holling (Eds.), Island Press, Washington, DC, 2002. Ecological Economics, 49(4), 488-491.

https://doi.org/10.1016/j.ecolecon.2004.01.010.

Price, M. F. (2002). Panarchy: understanding transformations in human and natural systems: edited by Lance H. Gunderson and C.S. Holling. Island Press, 2002. xxiv+507 pages. Biological Conservation, 114(2), 308-309.

https://doi.org/10.1016/S0006-3207(03)00041-7.

Smit, B., \& Wandel, J. (2006). Adaptation, adaptive capacity and vulnerability. Global Environmental Change, 16(3), $282-292$. https://doi.org/10.1016/j.gloenvcha.2006.03.008.

Turner, B. L., 2nd, Kasperson, R. E., Matson, P. A., McCarthy, J. J., Corell, R. W., Christensen, L., Eckley, N., Kasperson, J. X., Luers, A., Martello, M. L., Polsky, C., Pulsipher, A., \& Schiller, A. (2003a). A framework for vulnerability analysis in sustainability science. Proceedings of the National Academy of Sciences of the United States of America, 100(14), 8074-8079. http://dx.doi.org/10.1073/pnas.1231335100. PMid:12792023.

Turner, B. L., 2nd, Matson, P. A., McCarthy, J. J., Corell, R. W., Christensen, L., Eckley, N., Hovelsrud-Broda, G. K., Kasperson, J. X., Kasperson, R. E., Luers, A., Martello, M. L., Mathiesen, S., Naylor, R., Polsky, C., Pulsipher, A., Schiller, A., Selin, H., \& Tyler, N. (2003b). Illustrating the coupled human-environment system for vulnerability analysis: three case studies. Proceedings of the National Academy of Sciences of the United States of America, 100(14), 8080-8085.

http://dx.doi.org/10.1073/pnas.1231334100. PMid:12815106.

United Nations - UN. Department of Economic and Social Affairs Population Division. (2014a). World Urbanization Prospects: the 2014 revision. New York: United Nations. Recuperado em 20 de abril de 2015, de https://esa.un.org/unpd/wup/publications/files/wup2014-highlights.pdf

United Nations - UN. Department of Economic and Social Affairs Population Division. (2014b). World Urbanization Prospects: the 2014 revision (pp. 517). New York: United Nations. Recuperado em 20 de abril de 2015, de https://esa.un.org/unpd/wup/publications/files/wup2014-report.pdf

Van der Leeuw, S. E. (2001). 'Vulnerability' and the integrated study of socio-natural phenomena. IHDP Update, 2, 6-7. Recuperado em 20 de abril de 2015, de http://www.ihdp.unu.edu/docs/Publications/Secretariat/UpdateDimensions/Update-2-2001.pdf

Walker, B., Holling, C. S., Carpenter, S. R., \& Kinzig, A. (2004). Resilience, adaptability and transformability in social ecological systems. Ecology and Society, 9(2), 5. http://dx.doi.org/10.5751/ES-00650-090205.

Young, O. R., Berkhout, F., Gallopin, G. C., Janssen, M. A., Ostrom, E., \& Van Der Leeuw, S. (2006). The globalization of socioecological systems: an agenda for scientific research. Global Environmental Change, 16(3), 304-316. https://doi.org/10.1016/j.gloenvcha.2006.03.004.

\section{Editor: Harry Bollmann}

Recebido: Abr. 2, 2018

Aprovado: Ago. 28, 2018 